\title{
Description d'espèces de Dorcadion d'Espagne
} par

\author{
A. Chevrolat à Paris.
}

$\mathbf{P}$

rothorax offrant une côte longitudinale lisse, quelquefois uni-sillonnée. 1-15.

a. Antennes et pattes noires. $1-12$.

b. Antennes à $1^{\text {er }}$ art. et pattes rougeâtres. $13-15$.

Prothorax sans côte longitudinale. 16-29.

Espèces à corps noir. 1, 27-29.

Espèces à antennes noires. 1, 2, 4, 5-9, 13-17, 25-29.

Espèces à antennes maculées ou annelées de blanc ou de cendré. $3,8,10-12,18,20,23,24$.

Elytres sans lignes de couleur. 1, 22-27.

Elytres à lignes blanches ou cendrées ou brunes. 2-10, 1216, 18, 19.

Elytres avec la suture et la marge seules blanches. 20.

Elytres blanchâtres à côtes noires. 11 .

Elytres cendrées à côtes. 17.

1. Dorcadion encaustum. Elongatum, nigerrimum, nitidum, immaculatum; capite convexo, creberrime punctato, sulco longitudinali angusto ad verticem paululum utrinque reflexo; antennis atris, breviter pilosis et minute punctatis; prothorace transverso, crebre punctato, costâ longitudinali mediâ irregulari glabra, anguste sulcata, spina laterali acuta; elytris ovalibus vage et minute punctatis, minutissime coriaceis, singulatim anguste rotundatis et bicostatis; corpore infra pedibusque nitidis, minute punctatis, plantis tarsorum flavis. - Long. 15, Lat. 6 Millm. Hispania (Gallaecia).

Unique. Collect. de M. I. Reiche. 
Il se peut que cet exemplaire ait eu son indumentation effacée, toujours est il qu'aucune trace n'apparait et que je n'ai pu le rapporter à aucune autre espèce de ce groupe, la ponctuation et la disposition des côtés étant toute autre.

2. Dorcadion Ghilianii. Affine D. Perezi sed majus, nigrum, nitidum; capite crebre punctato, in vertice costis duabus glabris intus unisulcatis lineola alba antecessis; prothorace albo, lineis tribus nigris, media elevata glabra obsulcata, spina laterali brevi subobtusa; elytris ovalibus, singulatim bicostatis limbo marginali et suturali ad apicem anguste albis; albis quoque spatio a basi elytrorum intra costam internam usque ante apicem, lineola humerali integra (parte interiore elevata et valide punctata); pectore cinereo; abdomine nigro minutissime granulato et coriaceo, ano pedibusque infra cinereo limbatis. ㅇ. - Long. 15, Lat. 6 Millm.

Castille. de la Coll. de M. L. Reiche.

Cet insecte n'est peut-être que la 오 du D. Reichei $\sigma^{\nearrow}$; chez ce dernier le $1^{\mathrm{er}}$ article des antennes et les pattes sont rougeâtres tandisque ces organes sont entièrement noires chez le premier.

3. Dorcadion Perezi. Caput vittis tribus albidis, antennis nigris, thorace carina dorsali albido tenuissime limbata, lateribus canescente nigroque trivitlatis; scutellum nigrum nitidum; elytra sutura vittaque marginali alba, in apice uncinata, macula sagittiformi suturali conjuncta. - Long. 14, Lat. 5 Millm.

Dorcadion Perezi Ann. de la Soc. Ent. de Fr. 1851. p. 24. pl. 1. f. 8. in Guérin Rev. et Mag. de Zool. 1849. p. 620

Hispania (Guadarrama); Graëlls.

$ð$ et 오 de la Coll. de l'auteur.

4. Dorcadion alternatum. Nigrum, nitidum; capite vage punctato in medio fulvo alboque vario, longitudine sulcato, duabus costis frontalibus glabris, medio sulcatis; antennis nigris; prothorace angulose spinoso costis tribus glabris, lateralibus antice abbreviatis, elongato-quadratis, media basi depressa, secundum longitudinem foveata, obsolete alba et punctata; elytris ovalibus, holosericeis nigris, in singulis lineis quinque: quatuor albis (suturali, marginali et humerali integris altera basali abbreviata angusta) quinta mediana cinerea; corpore infra pedibusque nigris, nitidis, cinereo indutis, vage et minute punctatis. - Long. $12-13$, Lat. $3 \frac{1}{3}-6$ Millm.

Hispania (Guadarrama); D. Graëlls. 
Var. elytrorum linea fusca nulla.

오 $\sigma^{\nearrow}$ de la Coll. de l'auteur et de celle de M. Reiche.

J'avais désigné comme espèce sous le nom de $\boldsymbol{D}$. Costicolle une variété un peu plus alongée et étroite, d'un beau noir velouté, dont les elytres offrent une étroite bordure suturale, marginale et humerale d'un très beau blanc, mais chez laquelle la ligne fauve alterne fait défaut.

5. Dorcadion hispanicum. ठ․ Corps noir. Tête ornée, au moins à partir du milieu du front, de deux bandes d'un duvet blanc, prolongées sur le prothorax de chaque côté de la ligne mediane, celle-ci elevée lisse, luisante, sillonnée au milieu. Elytres offrant chacune deux bandes longitudinales d'un duvet blanc, couvrant la moitié interne de la largeure, à peine séparées entr'elles et de la suture, l'externe, postérieurement rétrécie et raccourcie et d'une bande semblable courte, oblique, ne couvrant que le quart postérieur de la longueur; le sommet de la marge est etroitement bordé de blanc. - Long. $11 \frac{3}{4}-12 \frac{1}{3}$, Lat. $4 \frac{1}{3}$ Millm.

ㅇ. Elytris laevibus convexis, indumento albo dense tectis, ad marginem nigris, nitidis, inaequaliter atque sat fortiter punctato-impressis, singulatim intus nigro-trilineatis, lineis angustis, duabus integris, intermedia ante apicem terminata et fere externae juncta.

Dorc. hispanicum Muls. opusc. II. p. 108. 1853. $\sigma^{\nearrow}$ (nec Dejean). $\sigma$ de la Coll. de l'auteur. $\sigma^{\nearrow}$ et $q$ de celle de M. Javet.

6. Dorcadion nigrolineatum. Breviter nigro-hirsutum, oblongum, nigrum, nitidum, inaequaliter minute vel ruge punctatum; in capite costâ longitudinali glabra, medio sulcata utroque albo marginata; prothorace aspere punctato, costâ longitudinali elevata glabra, medio sulcata, spinâ laterali brevi subacutâ; scutello albo; elytris ad apicem paululum ampliatis, in dimidio interno plusculum longitudinis albis, nigro-punctulatis et pilosis, in singulo lineis tribus angustissimis nigris postice conjunctis, ad partem externam basi nigris, ordine tuberculatis, dein punctatis; corpore infra pedibusque nigro-nitidis, minute punctatis, tibiis posticis cyanescentibus. 우 Long. 13, Lat. $5 \frac{3}{4}$ Millm.

Hispania; D. Ch. Ott. Unique. Coll. de l'auteur.

7. Dorcadion segovianum. Ђ゙. Oblongum, nigro-sericeum; capite convexo, nitido, antice passim, postice fortiter punctato, sulco 
longitudinali in vertice anguste bicostato, antennis omnino nigris; prothorace lateribus longe angulato et postice emarginato, ad medium bicostato (singulà costâ elevatâ glabrâ et nitidâ) et uni - sulcato, utrinque linea albida marginato; scutello albo, lineâ medià fuscâ; elytris ovalibus, nigro-brunneis, holosericeis, in singulo lineis quatuor albis, tribus integris, suturali et humerali latis (secundum ultimam altera lineola obsoleta cinerea ante apicem evanescente) marginali angusta, apice junctis, quarta in medio basis obliqua et parva; corpore infra pedibusque nigris, nitidis, inaequaliter punctatis, interstitiis minutissime coriaceis parceque cinereo-pilosis. - Long. 13, Lat. 5 Millm.

Hispania (Escurial); D. Graëlls.

Unique. Coll. de l'auteur.

Cette espèce est très voisine du D. Dejeanii. Elle s'en distingue par les caractères suivants: elytres un peu plus courtes, plus largement ovalaires d'un noir velouté à lignes plus larges d'un blanc pur, ligne humerale un peu plus rapprochée de la tranche externe; antennes noires, et non annelées de gris; côte longitudinale du prothorax large, elevée uni-sillonnée au milieu.

8. Dorcadion Dejeanii. Elongatum, nigro-tomentosum, opacum; capite convexo, nitido, minute et sat crebre punctato, sulco longitudinali utrinque albo-limbato; antennis sat validis nigris, ad basin articulorum albo-annulatis; prothorace inaequali subangulose et obtuse spinoso, duabus costis longitudinalibus mediis glabris et nitidis, intus sulcatis, utrinque albo-lineatis, margine laterali leucophaeo; scutello albo, lineâ nigra; elytris ob-ovalibus, nigro-sericeis, in singulis lineis quatuor albis: suturali, humerali latis et marginali angusta, omnino integris, quarta ad mediam basin incipiente, sed in $\sigma^{\top}$ ultra medium et in + tantum versus medium projecta; corpore infra pedibusque nigris, nitidis, tenuiter cinereo tectis, minute punctatis, et in interstitiis minutissime coriaceis. - Long. 13-15, Lat. $4 \frac{1}{2}-5$ Millm.

Dorcadion hispanicum Dej. Cat. 3. ed. p. 372. (nec Muls.)

Hispania; ex Museo Dejeanii.

$\delta$ et ㅇ de la Coll. de l'auteur.

Obs. La côte longitudinale du prothorax est étroite, chez le $\delta$ cette côte est faiblement sillonnée au milieu et ce sillon n'existe pas chez le 오. 
9. Dorcadion circumcinctum. Holosericeum, atro-opacum; in vertice capitis lineolis tribus albis (inter quas duabus nigerrimis) medianâ paululum protensâ atque sulcatâ; antennis basi sat latis nigris, articulis sex ultimis opacis; in prothorace costa longitudinali glabra, obsulcata, albo-limbata, spina laterali media, brevi, subreflexa; elytris ovalibus, convexis, holosericeis, nigro-opacis, omnino albo-circumdatis, versus medium basin breviter et oblique albo-unilineolatis; corpore infra pedibusque lanugine cinerea indutis, minutissime coriaceis, femoribus sparsim punctulatis; plantis tarsorum fulvis; pygidio cinereo. ‥ - Long. 8, Lat. 5 Millm.

Vieille Castille; de la Coll. de M. Linder.

10. Dorcadion Ariasi. ऽ. Ovale, nigro-opacum, sericeum; capite convexo, cinereo, anguste sulcato, duabus lineolis frontalibus nigris; palpis, mandibulis oculisque nigris; antennis crassis nigrobrunneo-opacis. Articulo primo et tertio basi griseis; prothorace cinereo, lineis quatuor albis, sulco longitudinali utrinque anguste elevato et glabro, extus angulose spinoso; scutello triangulari albo, lineâ mediâ nigrâ ; in singulo elytro lineis quatuor: tribus albis; suturali et marginali integris apice junctis; tertia basi abbreviata; quarta humerali integra fusca; corpore infra murino, minutissime coriaceo et pubescente.

ㅇ paululum ampla, convexa, praecipue differt: capite nigro, anguste sulcato alboque lineato, maculis $2^{\text {bus }}$ frontalibus brunneis extus albis; prothorace fusco; in singulo elytro tantum lineis $3^{\text {bus }}$ al-

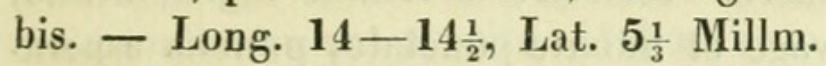

Hispania (Gallaecia).

Des Collect. de Mr. Reiche et de Mr. Arias Teijero, à qui je la dédie comme un faible témoignage de ma gratitude envers lui.

11. Dorcadion albicans. Breve, subelongatum, albidum; antennis nigris, albo-annulatis; in capite sulco longitudinali angusto nigro, in prothorace lineis tribus nigris: mediana elevata, angusta, nitida, tenui sulcata, in singulo elytro lineis tribus nigris: laterali lata et punctata, humerali lineari obsoleta, interna polita praecedenti ad apicem juncta; pedibus $\sigma^{\top}$ paululum denudatis; corpore infra pedibusque + indumento obscuro tectis; angulo prothoracis angulose spinoso, postea lateribus emarginato. Long. 10-11, Lat. 3-5 Millim.

Dorcadion albicans Dej. Cat. 3. ed. p. 372. types.

Hispania; ex mus. Dejeanii.

б et 우 de la Coll. de l'auteur. 
12. Dorcadion molitor. Thorace subspinoso, fuscus; vittis tribus integris albis, antennis mediocribus. - Long. 13-16, Lat. $5-6 \frac{1}{2}$ Millm.

Lamia molitor Fab. Syst. Ent. 1775. p. 176. 28.

Cerambyx molitor Ol. Ent. 1795. t. 4. No. 67. p. 115.154. pl.4. f. 23. lineola Ill. Mag. V. p. 238. 115.

$\sigma^{\nearrow}$ Coll. de Mr. Gougelet, 오 de celle de l'auteur. Cette dernière rapportée d'Espagne par notre Président Honoraire M. Leon Dufour qui lui avait imposé alors le nom de $\boldsymbol{D}$. lemniscatum diffère des exemplaires de la france meridionale en ce que les elytres sont maculés de petits taches d'un brun noirâtre.

13. Dorcadion longipenne. Elongatum nigrum; primo articulo antennarum, femoribus tibiisque ferrugineis, tarsis cinereis, articulo ultimo unguiculisque nigris; capite vage punctato, convexo, tomento fusco et albido in parte centrali variegato, costa longitudinali antice angusta, postice latiore glabra et nitida, intus sulcata, prothorace costis tribus glabris et nitidis, costâ medianâ integrâ; utrinque albo-limbata, costis lateralibus mediis, antice posticeque abbreviatis, extus late anguloso; scutello lato, subtriangulari, albo, lineâ longitudinali nigra, glabra; elytris longis, fuscis, singulo lineis quinque albis fere aequaliter distantibus: suturali, humerali et laterali integris, secunda interna posita, pone apicem limitata, et tertia infra basin incipiente, ultra medium protensa amboque angustis; prothorace infra et pectore cinereo-sericeis; abdomine nigro, nitido, brevissime cinereo vestito, vage et minute punctato, punctis asperatis tuberculiformibus, interstitiis minutissime coriaceis. - Long. 19 $\frac{1}{3}$, Lat. 5 Millm.

Hispania (Segovia?); D. Graëlls.

Unique. Coll. de l'auteur.

14. Dorcadion Reichei. Nigrum, subnitidum, partito opacum; antennis crassis, nigris, primo articulo antennarum, totis femoribus tibiisque anticis obscure rufis; capite aspere punctato, lineâ mediâ longitudinali limboque infero oculorum albis, sulco longitudinali angusto; prothorace nigro, opaco ruge et aspere punctato, lineis quatuor angustis albis ( $2^{\text {bus }}$ mediis et una in utroque latere), costâ mediâ angustâ glabrâ, antice paululum sulcata, angulo laterali subacuto; scutello nigro nitido, lateribus albis; elytris ovalibus, nigris, subnitidis, remote punctatis, singulo lineis $2^{\text {bus }}$ albis: una secundum suturam (sutura elevata nigra), secunda totam marginem epipleurisque 
tegente cum limbo externo angustissime nigro, costa humerali versus apicem suturae ducta; prothorace infra pectoreque cinereis, abdomine nigro-nitido, punctulato, ultimo segmento cinereo. - Long.

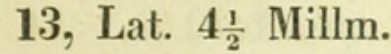

\section{Hispania.}

Unique. De la Coll. de Mr. Reiche, mon ami et ancien collègue, à qui je l'ai dédie comme souvenir de nos bonnes et agréables communications entomologiques. M. Thomson posséde également cette espèce.

15. Dorcadion Graëllsii (Chevr.). Tomento corporis destitutum nigrum (pedibus aliquando testaceis) nitidum; capite, thorace basique elytrorum punctato-scabris; fronte inter antennas, capite thoraceque, lineâ longitudinali elevatâ, medio sulcatâ et valde politâ praeditis; thorace ad latera inter lineam mediam et tuberculos coniformes, spatio elevato-oblongo et polito instructo; lineae elevatae praedictae et haec spatia fere semper depilata sunt. - Long. 12-15, Lat. 4-6 Millm.

Dorcadion Graëllsii (Chevr.) Graëlls Memorias de la Comision del mapa Geológico de España 1858. p. 91. Lam. V. sqq.

Hispania (Escurial, La Granja), Mai, Juni.

ð

Les elytres de cette espèce sont en ovale étroite, la suture, le bord marginal et une ligne humerale entière, réunies loutes trois au sommet, sont étroites et d'un beau blanc; entre cette dernière et la suture apparaissent deux autres lignes obsolètes, qui s'evanouissent insensiblement et dont l'interne seule est evidente à la base; leur intervalle est sillonné jusqu'au milieu longitudinal. Les antennes sont noires et le premier article est rougeâtre. (A. Chevr.)

16. Dorcadion navaricum. Noir, prothorax glabre, couvert de points gros et contigus, séparés par des intervalles rugueux et imponctués. Ecusson garni de poils blancs peu épais, isolés des bords latéraux et de la ligne médiane. Elytres revêtues d'un duvet velouté noir ou d'un noir brun, parées chacune d'une bordure suturale, d'une bordure externe à peu près d'égale largeur couvrant le rebord, d'une autre joignant ce dernier et plus étroite que lui, et de deux lignes longitudinales naissant de la base, d'un duvet blanc; l'externe, humérale, à peine plus large que la suturale, uniforme, prolongée presque jusqu'à l'extrémité, isolée de la bordure 
externe; l'autre, dépassant à peine les $\frac{2}{5}$ es. - Long. 15-17, Lat. $5 \frac{1}{2}-7 \frac{1}{2}$ Millm.

Dorcadion navaricum Dej. Cat. 3. ed. p. 372.; Muls. Opusc. II. p. 29. 1853.

Hispania (Navarre, Hautes pyrenées).

17. Dorcadion Seoanei. Nigro-opacum, subtilissime cinereo-pubescens; capite cicatricoso-punctato, fronte planulatâ, verticali in medio subsulcatâ et in centro sulci linea tenuissimâ impressa; mandibulis in basi nigro-setulosis; labro etiam nigro-setuloso et in margine rufo-ciliato, palpis laevibus; antennis minutissime punctatis in articulis primis frequenter depilatis, in ultimis pube brevissimâ et murino-obscurâ obtutis; prothorace supra scabroso profunde puntato-cicatricoso, antea et postea tenuiter marginato, marginibus brevissime ciliatis, medio lineâ deletâ vel vix signatâ subtus ante pectore subrugoso-punctato; scutello trigono transverso; elytris granulatione oblongâ et diversimode confluente asperatis, tricostatis, costis prominentibus, latis, asperis, parallelis, versus apicem evanescentibus, interstitiis latis, sulciformibus et griseo-cinerascentibus, brevissime setuloso-pilosis; pectore, abdomine pedibusque subglabris, pilis raris fere inconspicuis, rufulisque adspersis; punctuatione inaequali sublaevigata; tibiis in extremitate tarsiana setuloso-velutinis, uti dens intermediarum; pulvillis tarsorum fusco-ferrugineis. Long.

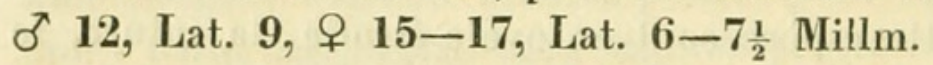

Dorcadion Seoanei Graëll's Mém. d. l. comis. del mapa Geol. de España 1858. p. 93.

Dorcadion sulcatum Dej. Cat. 3. éd. p. 372.

Hispania, in provincia de Leon (Puerto-Mauzonal).

o type unique. de la Coll. de l'auteur.

18. Dorcadion Handschuchii. Elongatum, elytris subparallelis; cinereo - tomentosum, capite thoraceque pallide ferrugineotinctis; elytris umbrinis, singulo fasciis tribus suturaque cinereis, margine laterali albo; antennis nigris, articulis basi cinereo-annulatis. - б Long. 8-9, Lat. $2 \frac{1}{2}-2 \frac{1}{4}$, क Long. 16, Lat. $6 \frac{1}{2}$ lin.

Dorcadion Handschuchii Küster Käfer Europ. XV. 77.

Hispania (Carthagène).

Unique. de la Coll. de M. J. Thomson.

19. Dorcadion quadrilineatum. Atrum, subtilissime cano pubescens, capite thoraceque punctatissimis; elytris oblongo-ovatis, 
fusco- tomentosis, sutura, margine inflexo vittisque duabus albidis,

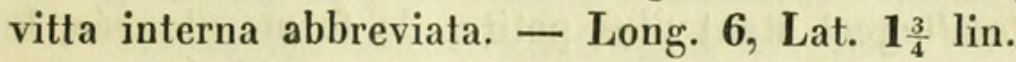

Dorcadion quadrilineatum Sturm Cat. 1843. p. 259; Küster Käfer Europ. VIII, 82.

Hispania.

Cette espèce, que je n'ai pas vue, parait être voisine de notre D. Suturale, mais chez ce dernier la marge n'est pas totalement blanche et n'a quelquefois de cette couleur que le sommet.

20. Dorcadion suturale. Validum, breviter oblongum, cinereum, tomento nigro-brunneo vestitum; antennis crassis brunneis, ad basin articulorum cinereo-annulatis; capite lato, convexo, griseoobscuro, macula magna occipitali cinerea, vage punctato, sulco longitudinali tenui; prothorace transverso, inaequali, medio foveato, punctis concutis et impressis signato, sulcis tribus transversis, primo lato secundum marginem anticum, tertio ante marginem posticum tertioque laterali supra praecedentem, spina laterali brevi acuta; scutello cinereo, basi semicircuiter impresso et fusco; elytris ovalibus, convexis, singulatim rotundatis, breviter hiantibus in sutura, bicostatis, obsolete fortiterque punctatis, tomento brunneo-obscuro cinereo parce nebulosis, in sutura et in margine postico anguste albo-lineatis. Corpore infra griseo-nitenti pube brevi et tenui denso vestito, punctulato, interstitiis creberrime et minute coriaceis; pedibus crassis, subtiliter parce adspersis, tibiis posticis extus arcuatis. $\delta^{7}$ et

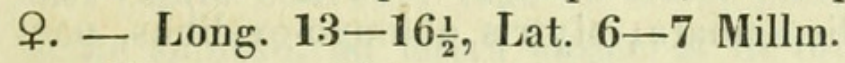

Hispania. Valence.

Des Collections de MMr. Reiche, Thomson, Javet et de la mienne. Cette espèce généralement envoyée d'Espagne sous le nom $\boldsymbol{D}$. mus Rshr. la diagnose qu'en donne l'auteur ne peut convenir, car il n'eut pas omis d'indiquer que la suture était blanche et j'ai reconnu dans le D. murinum Dej. l'insecte dont il s'agit; dans les individues $\sigma$, très frais, l'extrémité de la marge est aussi blanche.

21. Dorcadion Staudingeri. ठ大 Elongatum, fusco-brunneum, nitidum, partito minute concrete et vage punctatum; singulo elytro obtuse bicostato, suturâ tenui albâ; antennis sat validis, unicoloribus, nitidis, articulis tribus ultimis opacis et attenuatis; in capite sulco longitudinali paululum impresso; prothorace transverso, modice convexo, lateribus angulato et breviter spinoso. - Long. 13, Lat. 4 Millm.

Andalusia. 
J'avais d'abord pris cet insecte comme $\sigma^{\top} \mathrm{du} \boldsymbol{D}$. Suturale. Mais la taille du $\boldsymbol{D}$. Staudingeri étant plus petite, de forme alongée, le brillant du corps, la ponctuation fine, la couleur uniforme et plus particulièrement l'epaisseur moindre des antennes et l'absence d'indumentation noire, brune et cendrée aux elytres; ces nombreux caractères m'ont décidé à la séparer.

Coll. de MMr. L. Reiche et Kraatz.

22. Dorcadion Amorii. Nigrum nitidum, pube grisea vestitum, supra parce punctatum; fronte sulcata; pronoto utrinque obtuse dentato; elytris tenuiter bicostatis oblongo ovalibus, apice singulatim rotundatis; tibiis apice bispinosis, intermediis extus obtuse dentatis, fulvo-hirtis. - Long. 29, Lat. 10 Millm.

Dorcadion Amorii de Marseul Rev. et Mag. de Zool. Janv. 1856. p. 48.

Hispania; Sierra Morena.

Ce sera probablement l'une des plus grandes espèces du genre d'après un dessin fait par l'auteur; elle devra être placée près du D. annulicorne.

23. Dorcadion annulicorne. Elongatum, fuliginosum, opacum; corpore infra pedibusque cinereis; antennis brunneis albo-annulatis; capite convexo prothoraceque (spinâ laterali obtusa, in medio longitudinis lineola centrali laevi) crebre et ruge punctatis; scutello triangulari pilis luteolis limbato; elytris oblongo-ovalibus, paululum tomentosis, vage punctatis, conjunctim rotundatis; limbo postico segmentorum abdominalium nitido. $\delta$ ㅇ․ - 오 Long. 20, Lat.

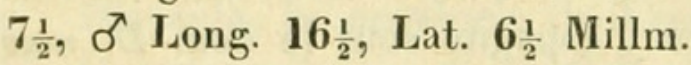

Hispania (Andalusia).

Cette grande et belle espèce s'éloigne de la forme ordinaire et rappele celle de la Lamia textor; la 우 fait partie de la collection de notre ami et savant collègue Mr. Reiche et le $\sigma^{\top}$ m'a été communiqué par Mr. le Dr. Kraatz.

24. Dorcadion lusitanicum. Oblongum, nitidum, fuliginosum, vage punctatum; capite fortiter et ruge punctato, longitudine sulcato, obtuse et fere bicornuto, mandibulis lucidis; thorace inaequali, in disco costulâ longitudinali abbreviata, mediâ, dente acuto laterali; elytris modice elongato-ovalibus et convexis, singulatim apice

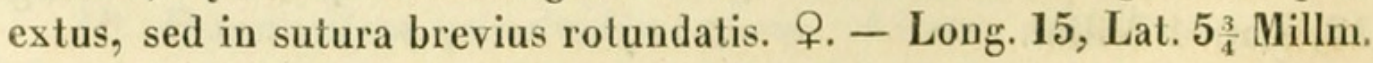

Lusitania. 
ð Elongatus; elytris pube brunnea, vage et seriatim maculatis.

Dorcadion lusitanicum Chevr. Rev. Zool. par la Soc. Cuvier. Ann. 1840. p. 16. No. 18. ㅇ.

Type + de la Coll. de l'auteur; $ð$ de celle de M. J. Thomson.

Cette espèce me paraît se rapprocher beaucoup du D. mus Rosenh. mais les antennes du notre sont plus longues, amincies au sommet et les premiers articles sont moins epais; elle se rapproche aussi du $\boldsymbol{D}$. Staudingeri, mais ce dernièr a 2 petites côtes à chaque étui et la suture est blanche, ce qui n'a pas lieu chez le $\boldsymbol{D}$. lusitanicum.

25. Dorcadion mus. Oblongo-ovatum, nigrum, opacum, flavescenti-cinereo-tomentosum, unicolor, sparsim punctatum; prothorace lateribus obtuse spinoso; scutello brevi, lato, apice rotundato

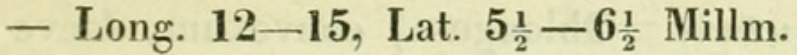

Dorcadion mus W. G. Rosenh. die Thiere Andalus. 1856. p. 304. Dorcadion murinum Dej. Cat. 3. ed. p. 372. types.

Andalusia.

ㅇ de la Coll. de l'auteur.

26. Dorcadion soricinum. Statura D. fuliginatori F., oblongum, nigrum; mandibulis, antennis brevibus, validis opacis, pedibus corporeque infra confertissime punctulatis coriaceis atque nitidis; capite convexo, vage et crasse punctato; prothorace transverso, acute spinoso, ad latera antica et in tota basi sulcato et marginato, punctis inaequalibus foveolatis impresso atque extus rugose asperato; scutello triangulari medio depresso; elytris oblongis, indumento griseo tutis, singulatim rotundatis a basi versus medium subremote punctatis, costula longitudinali interna ante medium abbreviata; tibiis intermediis versus apicem extus emarginatis nigro ciliatis et fere

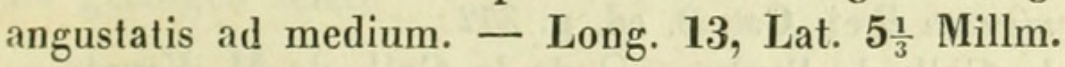

Hispania.

D. murinum Dej. Cat. 3. ed.

Dejean avait confondu, dans sa collection, deux espèces sous ce nom. La seconde, je l'ai rapportée au $\boldsymbol{D}$. mus de Rosenhauer.

27. Dorcadion castilianum. ठ Elongatum, totum nigrum, nitidum; capite antice sulcato, super insertionem antennarum breviter bicostato, profunde inaequaliter punctato, in interstitiis punctorum punctulato, punctis frontalibus majoribus paululum adstrictis et foveolato-reticulatis; antennis validis, elongatis, punctulatis; protho- 
race transverso, fortiter et reticulatim punctato, spina laterali brevi reflexa; scutello subtriangulari, postice rotunde producto, sulco basali transverso; elytris oblongo-elongatis, ab humero extus versus medium subobtuse carinatis, subseriatim punctatis, singulatim rotundatis et tricostatis, costa interna elevata, interstitiis costarum obsulcato-punctatis, epipleuris declivibus, sulcatis externe reflexis; limbo postico segmentorum abdominalium cineo-nitenti; pedibus lacertosis, tarsis latis. - Long. 18, Lat. 6 Millm.

Castille.

Cette belle espèce qui doit sans doute être placée près du $\boldsymbol{D}$. Amorii de Mars. m'a été communiquée par Mr. Henri de Bouvouloir, comme étant le D. Seaonei de Graëlls, mais la description de cet auteur ne lui convient pas.

28. Dorcadion Lorquinii. Oblongum, convexum, laeve sparsim punctatum nigro-brunneum, sat nitidum; antennis tibiis tarsisque brunneis; capite canaliculato; prothorace laevi, utrinque breviter spinoso; elytris convexis, laevigatis, sparsim et tenuissime punctatis, humeris fortius punctatis; sutura postice paulo elevata. Long. 14-15, Lat. 6-6 $\frac{1}{2}$ Millm.

Dorcadion Lorquinii Fairm. Ann. d. 1. Soc. Entom. d. Fr. 1855. p. 322 . Kraatz.

Hispania (Sierra Nevada); de la Collect. de l'auteur et de M.

29. Dorcadion Spinolae. Breve, aterrimum, nitidum, immaculatum, vertice thoraceque rugosis, elytris crebre punctatis sub-

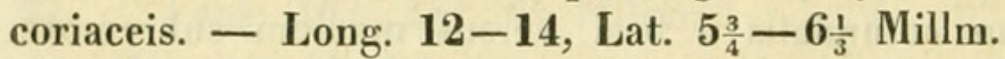

Lamia (Dorcadion) spinolae Dalm. in Sch. app. ad syn. p. 174. No. 241. types.

Dorcadion nigrum Dej. Cat. 3. ed. p. 372.

$\sigma^{\top}$ et $q$ de la Coll. de l'auteur, $q$ de la Coll. de M. Kraatz. 


\section{$2 \mathrm{BHL}$ Biodiversity Heritage Library}

Chevrolat, Auguste. 1862. "Description d'espèces de Dorcadion d'Espagne." Berliner entomologische Zeitschrift / herausgegeben von dem Entomologischen Vereine in Berlin 6, 337-348.

View This Item Online: https://www.biodiversitylibrary.org/item/37790

Permalink: https://www.biodiversitylibrary.org/partpdf/36662

\section{Holding Institution}

Smithsonian Libraries

\section{Sponsored by}

Smithsonian

\section{Copyright \& Reuse}

Copyright Status: Public domain. The BHL considers that this work is no longer under copyright protection.

This document was created from content at the Biodiversity Heritage Library, the world's largest open access digital library for biodiversity literature and archives. Visit BHL at https://www.biodiversitylibrary.org. 